Bull. Austral. Math. Soc.

VOL. 40 (1989) [231-234]

\title{
SOME GROUPS ALL OF WHOSE PROPER VERBAL QUOTIENTS ARE RELATIVELY FREE
}

\author{
Graham Higman
}

\section{For Bernhard Neumann with best wishes on his eightieth birthday}

\begin{abstract}
A class of blocking pairs $(A, B)$ for which $B / A^{B}$ is infinite cyclic is constructed and these are used to construct groups all of whose proper verbal quotients are relatively free.
\end{abstract}

\section{INTRODUCTION}

This note has three purposes. The first, of course, is to congratulate Bernhard on reaching this latest inilestone and to express the wish that in his next decade he may enjoy life and give pleasure to the rest of us as he always has.

The second purpose is to record that a conjecture 1 made in [4] is false. In that paper $I$ introduced the notion of a blocking pair (and for convenience the definition is repeated below) and $I$ conjectured that a group can be written as a quotient $B / A^{B}$, where $(A, B)$ is a blocking pair, if and only if it is countable, and locally free and has no infiuite cyclic free factor. It is trivial that $B / A^{B}$ is countable and that it is locally free is proved in [4], but $I$ was wrong to suppose that it can have no infinite cyclic free factor. In fact, in Section 2 below I construct a class of blocking pairs $(A, B)$ for which $B / A^{B}$ is itself infinite cyclic.

The third purpose of this note is the one alluded to in the title. The point of introducing blocking pairs is that they are an ingredient in a recipe for making countably free groups of cardinality $\omega_{1}$ which are not strongly countably free, and therefore not free. In Section $3 \mathrm{I}$ show that if some of the pairs constructed in Section 2 are used in this recipe, the group $G$ that emerges has the property that, for every non-trivial verbal functor $v$, the group $G / v(G)$ is relatively free of rank $\omega_{1}$ in the variety determined by $v=1$. Because it is countably free, $G$ is residually nilpotent and therefore parafree. Thus $G$ may be compared with parafree groups constructed by Gilbert Baumslag [1] (see also Dunwoody [3]), all of whose proper verbal quotients are relatively free of rank $r$, where $r$ is a fixed positive integer; and with the parafree group of Baumslag and Urs Stammbach [2], which is countably free of cardinality $\omega_{1}$ and all of whose lower central quotients are relatively free of rank $\omega$.

Received 11 April, 1989

Copyright Clearance Centre, Inc. Serial-fee code: 0004-9729/89 \$A2.00+0.00. 


\section{BLOCKING PAIRS WITH CYCLIC QUOTIENTS}

By definition, a blocking pair is a pair $(A, B)$ of groups such that

(i) each of $A, B$ is a free group of countably infinite rank;

(ii) $A$ is a proper subgroup of $B$, but is not contained in a proper free factor of it;

(iii) every finitely generated free factor of $A$ is a free factor of $B$.

Our object in this section is to construct a class of blocking pairs $(A, B)$ for which the factor group $B / A^{B}$ is infinite cyclic. For each natural number $r$ we define $A_{r}$ to be the free group freely generated by $a_{0}, a_{1}, \ldots, a_{r}$, and $B_{r}$ to be the free group freely generated by $a_{0}, a_{1}, \ldots, a_{r}, c_{r}$. For each $r$ we embed $B_{r}$ in $B_{r+1}$, by identifying $c_{r}$ with $w_{r}^{-1} c_{r+1} w_{r}$ where $w_{r}$ is an element of $\left\langle a_{r}, a_{r+1} c_{r+1}\right\rangle$ chosen to satisfy

$$
w_{r}^{-1} c_{r+1} w_{r} \text { does not belong to either of the double cosets } A_{r+1} c_{r+1}^{ \pm 1} A_{r+1} .
$$

There is no difficulty in finding such elements; any non-trivial element of the derived subgroup of $\left\langle a_{r}, a_{r+1} c_{r+1}\right\rangle$ will do. We then set $A=\bigcup_{r} A_{r}$ and $B=\bigcup_{r} B_{r}$, and aim to prove:

$$
(A, B) \text { is a blocking pair such that } B / A^{B} \text { is infinite cyclic. }
$$

Proof: Evidently $A$ is freely generated by $a_{0}, a_{1}, a_{2}, \ldots$ Notice next that since $w_{r}$ belongs to $\left\langle a_{r}, a_{r+1} c_{r+1}\right\rangle$ it belongs to $\left\langle B_{r}, a_{r+1} c_{r+1}\right\rangle$, whence so also do $c_{r+1}\left(=v_{r} c_{r} w_{r}^{-1}\right)$ and $a_{r+1}$. That is $B_{r+1}=\left\langle B_{r}, a_{r+1} c_{r+1}\right\rangle$. Since $B_{r+1}$ is a free group of finite rank one greater than the rank of $B_{r}$, this implies that $B_{r+1}$ is the free product of $B_{r}$ and $\left\langle a_{r+1} c_{r+1}\right\rangle$. Thus, for any $r, B$ is freely generated by $a_{0}, \ldots, a_{r}, c_{r}, a_{r+1} c_{r+1}, a_{r+2} c_{r+2}, \ldots$ From this it follows not only that $B$ is a free group of countably infinite rank, but also that every finitely generated free factor of $A$ is a free factor of $B$ (for it is a free factor of $A_{r}$ for some $r$ ).

Now let us denote images of elements of $B$ in the natural map of $B$ to $B / A^{B}$ by a bar. Since $B$ is generated by $a_{0}, a_{1}, \ldots, c_{0}, c_{1}, \ldots$ subject to the defining relations, $c_{r}=w_{r}^{-1} c_{r+1} w_{r}$ for $r=0,1,2, \ldots$, and the elements $a_{0}, a_{1}, \ldots$ generate $A, B / A^{B}$ is generated by $\bar{a}_{0}, \bar{a}_{1}, \ldots, \bar{c}_{0}, \bar{c}_{1}, \ldots$ subject to the delining relations $\bar{a}_{0}=\bar{a}_{1}=\ldots=1$, $\bar{c}_{r}=\bar{w}_{r}^{-1} \bar{c}_{r+1} \bar{w}_{r}$, for $r=0,1,2, \ldots$ But $w_{r}$ is an element of $\left\langle a_{r}, a_{r+1} c_{r+1}\right\rangle$, so that $\bar{w}_{r}$ is a power of $\bar{c}_{r+1}$, and the second block of relations becomes $\bar{c}_{0}=\bar{c}_{1}=\bar{c}_{2}=\ldots$ Thus $B / A^{B}$ is infinile cyclic, and, in particular, $A$ is a proper subgroup of $B$.

Thus to complete the proof of (2.2) we have only to show that $A$ is not contained in a proper free factor of $B$. Suppose then that $B$ is the free product $X \star Y$ where $A \leqslant X$ and $Y \neq 1$. Then $Y$ is a free group and is isomorphic to $B / X^{B}$, which is a homonorphic image of the cyclic group $B / A^{B}$. So $Y$ is cyclic, say $Y=\langle b\rangle$. 
Since $B=\bigcup_{r} B_{r}, b$ belongs to $B_{r}$ for large enough $r$. By the Kurosh sulgroup theorem $B_{r}=\left(X \cap B_{r}\right) \star\left(Y \cap B_{r}\right) \star Z$ for some group $Z$. Now $b$ belongs to $B_{r}$, so $Y \cap B_{r}=Y \neq 1$, so that $X \cap B_{r}$ is a proper free factor of $B_{r}$. But $X \cap B_{r}$ contains $A_{r}$ which is a free factor of $B_{r}$ of co-rank 1, hence a maximal proper free factor. It follows that $X \cap B_{r}=A_{r}$ and that $Z=1$. That is, $B_{r}$ is freely generated by $a_{0}, a_{1}, \ldots, a_{r}, b$ as well as by $a_{0}, a_{1}, \ldots, a_{r}, c_{r}$; which in turn implies that the double cosets $A_{r} b A_{r}$ and $A_{r} c_{r}^{ \pm 1} A_{r}$ are the same, for one or other choice of sign. But, of course, if $b \in B_{r}$ then certainly also $b \in B_{r+1}$, so that the same argurnent proves that $A_{r+1} b A_{r+1}$ coincides with one of $A_{r+1} c_{r+1}^{ \pm 1} A_{r+1}$. But then $c_{r}$ belongs to one or other of $A_{r+1} c_{r+1}^{ \pm 1} A_{r+1}$, whereas by (2.1) this is not so. This contradiction completes the proof of (2.2).

\section{NON-FREE GROUPS WITH RELATIVELY FREE VERBAL QUOTIENTS}

The blocking pairs used in this section will be constructed by the method of Section 2; but we shall need to impose a stronger condition than (2.1) on the choice of the elements $w_{r}$. Let $\left\{v_{i}, i=0,1, \ldots\right\}$ be a listing of the non-trivial elements of a free group $F$ of countable infinite rank, and assume that $v_{0}$ is a commutator. We shall use the symbols $v_{i}$ also to denote the corresponding verbal functors. That is, for any group $G, v_{i}(G)$ will denote the subgroup of $G$ generated by the images of $v_{i}$ under homomorphisms of $F$ into $G$. If $F_{2}$ is a free group of rank 2 then, of course, $v_{i}\left(F_{2}\right) \neq 1$ for any $i$, because $F_{2}$ contains an isomorphic copy of $F$; and $\bigcap_{i=0}^{r} v_{i}\left(F_{2}\right) \neq 1$, because the intersection of a finite set of non-trivial normal sulggroups of a free group is non-trivial. The stronger condition on the elements $w_{r}$ that we need is:

$$
w_{r} \text { is a non-trivial element of } \bigcap_{i=0}^{r} v_{i}\left(\left(a_{r}, a_{r+1} c_{r+1}\right)\right) .
$$

Because we have assumed that $v_{0}$ is a commutator, (3.1) implies (2.1). Then we have

(3.2) If the blocking pair $(A, B)$ is constructred as in Section 2, with elements $w_{r}$ satisfying (3.1), then, for any non-trivial verbal functor $v, B / v(B)$ is the free product of $A / v(A)$ and a free cyclic group, where the terms "free product" and "free cyclic" are to be interpreted in the variety $\mathcal{V}$ of groups $G$ satisfying $v(G)=1$.

Proof: $B / v(B)$ is the group generated in $\mathcal{V}$ by $a_{0}, a_{1}, \ldots, c_{0}, c_{1}, \ldots$, subject to the relations $c_{r}=w_{r}^{-1} c_{r+1} w_{r}$. But $v$ is non-trivial, so that, for some integer $i, v_{i}$ belongs to $v(F)$, whence $v_{i}(G) \leqslant v(G)$ for any group $G$. Thus, (3.1) implies that in $\mathcal{V}$ we have $w_{r}=1$ for $r \geqslant i$. Thus, the relations $c_{r}=w_{r}^{-1} c_{r+1} w_{r}$ for $r \geqslant i$ reduce to $c_{i}=c_{i+1}=c_{i+2}=\ldots$. If this element is denoted by $c$, the relations for $r<i$ simply 
define $c_{0}, \ldots, c_{i-1}$ in terms of $a_{0}, a_{1}, \ldots$ and $c$, so that $B / v(B)$ is free (in $\mathcal{V}$ ) on these generators. But $a_{0}, a_{1}, \ldots$ generale $A / v(A)$ in $\mathcal{V}$, so we are home.

Recall next, from [4], that a blocking tower is a tower $\left\{A_{\alpha}, \alpha<\omega_{1}\right\}$ of groups such that (i) for each finile or countable ordinal $\alpha,\left(A_{\alpha}, A_{\alpha+1}\right)$ is a blocking pair and (ii) the tower is smooth, that is, for limit ordinals $\alpha, A_{\alpha}=\bigcup_{\beta<\alpha} A_{\beta}$. The type sequence of a blocking tower is the sequence of length $\omega_{1}$ of isomorphism types of blocking pairs, in which the $\alpha$-th term is always the isomorphism type of $\left(A_{\alpha}, A_{\alpha+1}\right)$. It is proved in [4] that every sequence of length $\omega_{1}$ of isomorphism types of blocking pairs is the type sequence of a blocking tower, and that the union of the groups in a blocking tower is a countably free group of cardinality $\omega_{1}$ which is not strongly countably free. As in [4], if $(A, B)$ is a blocking pair, we shall write $G(A, B)$ for a group which is the union of the groups in a blocking tower whose type sequence is constant, being equal to the isomorphism type of $(A, B)$ for all $\alpha$. We shall prove:

(3.3) If $(A, B)$ is a blocking pair constructed by the method of Section 2 with elements $w_{r}$ satisfying (3.1) then $G=G(A, B)$ is a countably free group of cardinality $\omega_{1}$ which is not free, such that for every non-trivial verbal functor $v, G / v(G)$ is the free group of rank $\omega_{1}$ in the variety of groups $X$ satisfying $v(X)=1$.

ProOF: $1 t$ is part of the general theory that $G$ is countably free of cardinality $\omega_{1}$ but not strongly countably free. By assumption $G$ is the union of a smooth tower $\left\{A_{\alpha}, \alpha<\omega_{1}\right\}$ where $\left(A_{\alpha}, A_{\alpha+1}\right)$ is isomorphic to $(A, B)$. By Corollary 2.11 of [4] each $A_{\alpha}$ is a local retract of $G$ whence $v\left(A_{\alpha}\right)=v(G) \cap A_{\alpha}$, so that $A_{\alpha} / v\left(A_{\alpha}\right)$ embeds in $G / v(G)$ for each $\alpha$. Thus $G / v(G)$ is the union of the smooth tower of groups $\left\{A_{\alpha} / v\left(A_{\alpha}\right), \alpha<\omega_{1}\right\}$. Now $A_{0}$ is an absolutely free group of countable rank, so that $A_{0} / v\left(A_{0}\right)$ is free of countable rank in $\mathcal{V}$, freely generated, say, by the countable set $C$. By (3.2), for each $\alpha, A_{\alpha+1} / v\left(A_{\alpha+1}\right)$ is the free product in $\mathcal{V}$ of $A_{\alpha} / v\left(A_{\alpha}\right)$ and the $\mathcal{V}$-free cyclic group $\left\langle c_{\alpha}\right\rangle$. This, with smoothness, guarantees that $G / v(G)$ is freely generated in $\mathcal{V}$ by the set $C \cup\left\{c_{\alpha}, \alpha<\omega_{1}\right\}$. We have proved the result.

\section{References}

[1] Gilbert Baumslag, 'More groups that are just about free', Bull. Amer. Math. Soc. 74 (1968).

[2] Gilbert Baumslag and Urs Stammbach, 'A non-free parafree group all of whose countable subgroups are free', Math. Z. 148 (1976).

[3] M.J. Dunwoody, 'Review of [1], MR 37 \# 1449'.

[4] Gralıam Iligman, 'Some countably Iree groups', in Group Theory, Proceedings of the Singapore International Conference, Editors K.N. Clieng and Y.K. Leong (De Gruyter, 1989). 